\title{
Application of the Integration of the Internet of Things and Big Data
}

\author{
Yingli Wang a \\ Guangzhou Huali Science and Technology Vocational College, Guangzhou 511325, China. \\ a105624725@qq.com.
}

Keywords: big data; the Internet of Things; integration.

\begin{abstract}
In order to perfectly integrate the Internet of Things (IOT) and big data, the way how big data influences the construction of an open IOT ecosystem was discussed and the significance of big data for the Internet of Things was set forth. Also, the system framework of an open IOT ecosystem with advanced communication and analytics technologies and new applications generated after the integration of big data and the Internet of Things as well as the big data technology framework in the Internet of Things was discussed. The research results showed that the integration of big data and the Internet of Things was bound to bring the intelligence of human society to a new height. It can be seen that the application of integrating the Internet of Things into the big data is unlimited.
\end{abstract}

\section{Introduction}

As more and more people use easier connection equipment, the Internet of Things (IOT) continues to evolve. As a result, the availability of data is exponentially distributed [1]. How can we find truly valuable data through the large amount of information?

At present, the Internet of Things has been widely used, and the volume of big data is also growing exponentially. The integration of the Internet of Things and big data has become an inevitable trend in the development of various industries [2]. This article examines how big data will affect the Internet of Things and the application of their integration in our daily production and life. The advanced communication and analysis technologies in the Internet of Things include the foundation of the Internet of Things, the communication between devices and clouds in the Internet of Things, and the integration of the Internet of Things with telecom operators. New applications generated by the integration of big data and Internet of Things include an overview of big data applications and how big data affects IOT applications [3]. The big data technology framework in the Internet of Things and the advanced analysis protocol stack of the Internet of Things include data capture, data integration and management, big data technology framework, and data mining in the Internet of Things.

\section{Literature Review}

The Internet of Things (IOT) is called the third wave of the development of the world's information industry after the computer and the Internet. It is the integration of IntelliSense, recognition technology with pervasive computing, and ubiquitous networks [4]. The earliest practice of the Internet of Things can be traced back to Xerox's cyber Cola vending machine in 1990. The concept of the Internet of Things was first proposed by Professor Kevin Ashton of the Massachusetts Institute of Technology in 1999. The International Telecommunication Union officially elaborated on the concept of the Internet of things in its ITU Internet Report 2005: The Internet of Things — — The Internet of Things is a kind of network that can realize the true functions of accurate positioning, accurate identification, intelligent monitoring and management through radio frequency identification device (RFID) installed on objects, infrared sensor, global positioning system, laser scanners, and various sensors. It can conduct communication between any objects any time at any place.

So far, the development of Internet of Things technology has been very extensive. For example, the Internet of Things has been used in smart city, smart healthcare, intelligent transportation, smart 
home, intelligent agriculture and many other fields [5]. At present, China has made attempts in many fields, such as car networking, smart medical care, smart home etc. and has achieved good results [6].

What is big data anyway? Industry insiders have not given a unified definition even in book The Age of Big Data by Viktor Meyer Schonberg. With the rapid development of computer technology and Internet technology, modern society is generating big data at an unimaginable speed, such as mobile phone communications, website visits, microblog messages, video uploads, product generation, scientific experiments etc. The ubiquitous social and commercial activities are generating a steady stream of data. In order to understand the characteristics of big data more clearly, we will analyse big data from four dimensions: data volume, data velocity, data diversity, and data value, as shown in Figure 1.

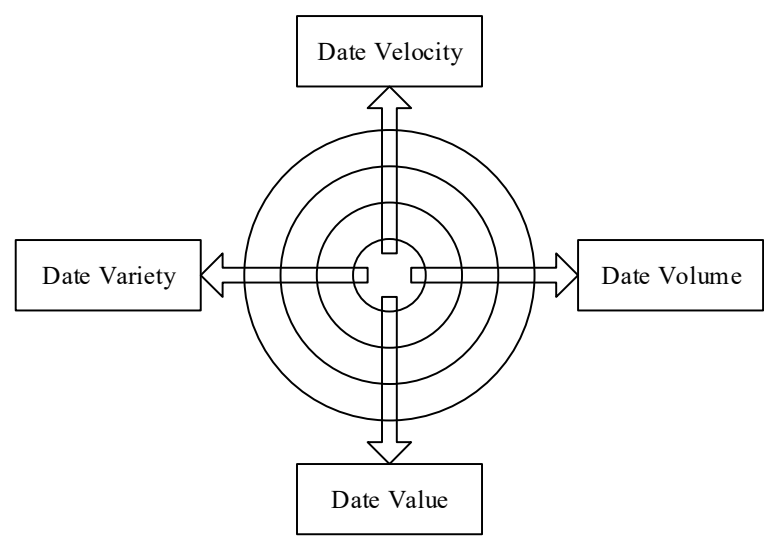

Figure 1. " $4 \mathrm{~V}$ " features of big data

\section{Methodology}

\subsection{The Architecture of ITU-T Internet of Things}

The Internet of Things is an important part of the new generation of information technology. Its English name is "The Internet of Things." As the name implies, the Internet of Things is the Internet that connects objects." This has two meanings: first, the core and foundation of the Internet of Things is still the Internet. And it is an expanded network based on the Internet; secondly, its client can extend to anywhere between any items and conduct information exchange and communication. At present, the definition of the Internet of Things can be referred to the relevant literature. Therefore, the Internet of Things is a kind of network that uses information sensing devices such as radio frequency identification, infrared sensors, global positioning systems, and laser scanners to connect any item to the Internet and perform information exchange and communication in order to achieve the functions of intelligent identification, positioning, tracking, monitoring and management of items.

The first Internet of Things standard developed by ITU-T is Internet of Things Overview. The Internet of Things is the global infrastructure of the information society and can connect objects to each other to provide advanced services based on existing and/or evolving interoperable information and communication technologies. The Internet of Things makes full use of items to provide services for various applications by using the capabilities of identification, data collection, processing, and communication. Meanwhile, it can ensure necessary privacy.

\subsection{Integration of Cloud Computing and the Internet of Things}

The communication between devices and the cloud in the Internet of Things is usually divided into four modes, namely, telemetry, inquiry, command, and notification. Telemetry refers to a client device sending data to a cloud device in one way. Query means that the cloud device sends a query notification to the client device, and the client makes a reply which says having received information successfully or not. Command means that the client sends a command to the cloud device and the client device makes a reply which says having received information successfully or not. Notification means that the cloud device sends a notification which is very important for the operation of the device to the client device. A series of applications that may be widely used or significantly improved in the future due to cloud computing and the Internet of Things are described, as shown in Figure 2. 


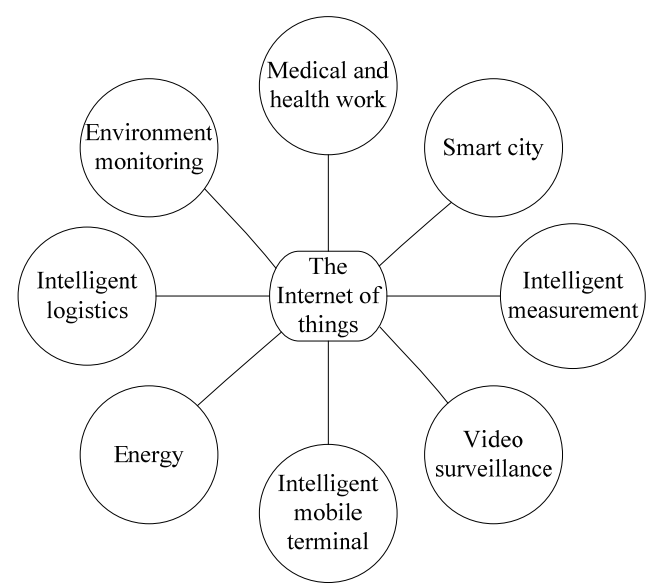

Figure 2. Application scenarios driven by the IOT paradigm

\section{Results and Discussion}

\subsection{New Applications Generated by the Integration of Big Data and Internet of Things}

Big data is a term used to describe any large number of structured, semi-structured, and unstructured data that may be tapped into information. PB-level data can be used as an example of big data, which consists of billions to trillions of records from different sources. Various types of data are usually loosely structured and are incomplete and inaccessible. Since a large amount of useful data and information can be extracted from an application, this is very beneficial. It can also save costs, increase the efficiency of information collection, and improve innovation capabilities, which can be expressed in Formula 1. Figure 1 also shows the application of the integration of large data and the Internet of things.

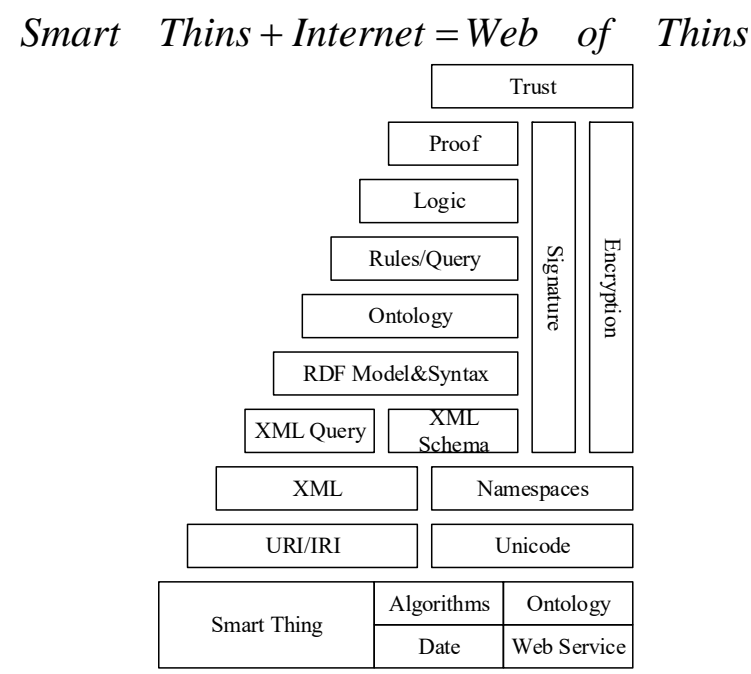

Figure 3. Integration of big data and the Internet of Things

Big data is mainly used in the medical and health industry, the network enterprises, advertising and marketing industry, and financial service industry. Various applications have depicted an application diagram for the Internet of Things. Big data will also affect the application of the Internet of Things. It may affect industries such as manufacturing, asset and fleet management, resource exploration, healthcare, retail, and logistics.

\subsection{Big Data Analysis Protocol Stack for the Internet of Things}

AMQP stands for Advanced Message Queuing Protocol, a message centre that originated in the banking middleware. It relies on queuing and publishes subscription messages based on topics. The AMQP model includes producers, exchanges, bindings, and queues. The message producer creates a message, fills it with content, gives the message a routing key, and then sends it to an exchange. The exchange accepts messages from the message producer and sends them to the message queue by route if the messages satisfy the binding standard of the expression. Binding is defined as the relationship 
between exchanges and message queues, specifying which message should be routed to a given queue. If the queue is not bound to the exchange, no message will be received from the exchange. A message queue stores the information and transmits it to the subscribed message consumer queue. Message consumers can create, subscribe, share, use, or destroy message queues. Figure 4 shows the schematic diagram of the AMQP protocol.

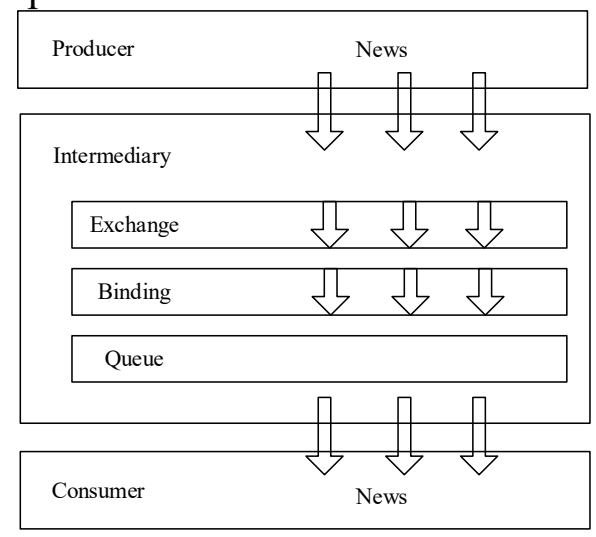

Figure 4. Schematic diagram of the AMQP protocol

COAP follows the client/server model. The client sends a request to the server and the server sends back a response. Clients can view, modify, create, and delete resources. The design of COAP can achieve interoperability with HTTP and REST through simple proxies, because COAP is based on datagrams and it can use SMS and other packet-based communication protocols on the top.

\subsection{Data Acquisition}

The two ends of M2M are connected to the machine and can only perform simple communication functions. Generally, it can only communicate between the equipment in an enterprise and the equipment in the industry chain and requires simple data processing capabilities. M2M technology has become a kind of ubiquitous new technology for communication between devices, and it is the most common application form of the Internet of Things at present. In the three-tier architecture of the Internet of Things, the M2M terminal is an important part of the perceptive extension layer. The M2M terminal is a device that connects the perceptive extension layer and the network layer. It mainly realizes the functions of data collection (or aggregation) and data transmission to the network. It's mainly responsible for data acquisition, pre-processing, encryption, control, and data transmission functions. As shown in Figure 5.

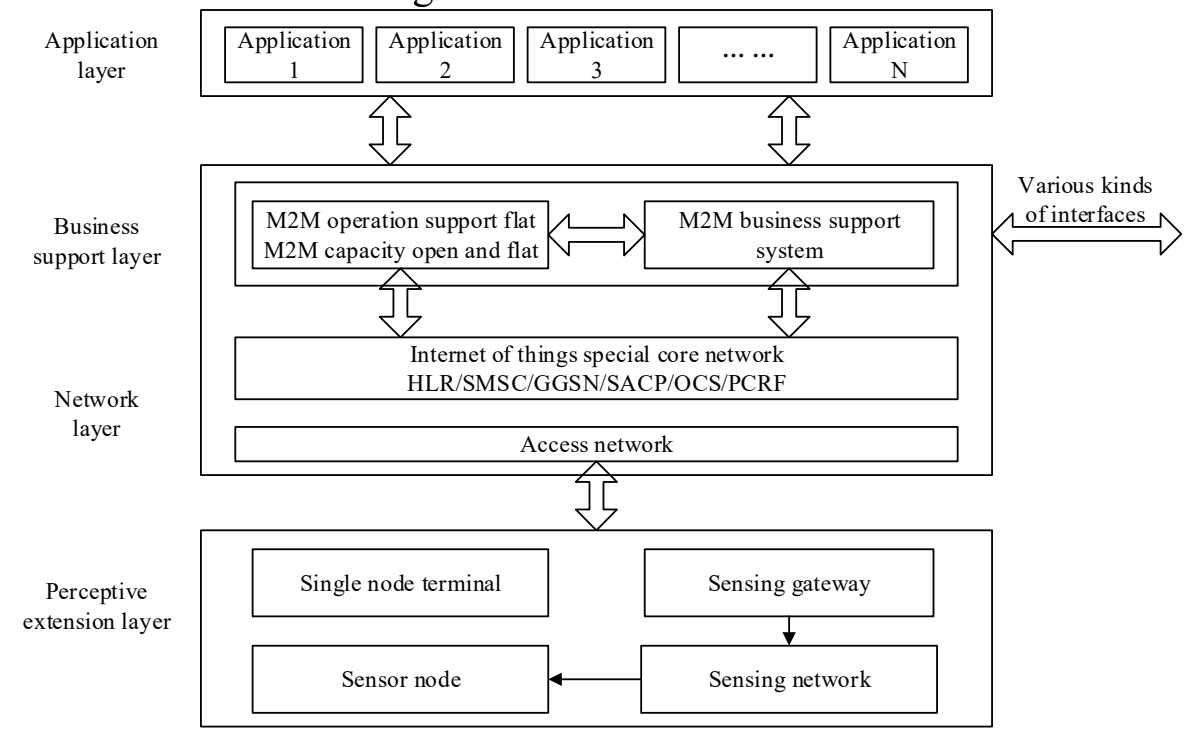

Figure 5. Architecture of the Internet of things

The functional modules of the terminal equipment of the Internet of Things mainly include a data acquisition and control module, a data convergence module, a core processing module, a telecommunication network access module, a peripheral interface module, and a power supply module. The functional structure of the Internet of Things terminal is shown in Figure 6. 


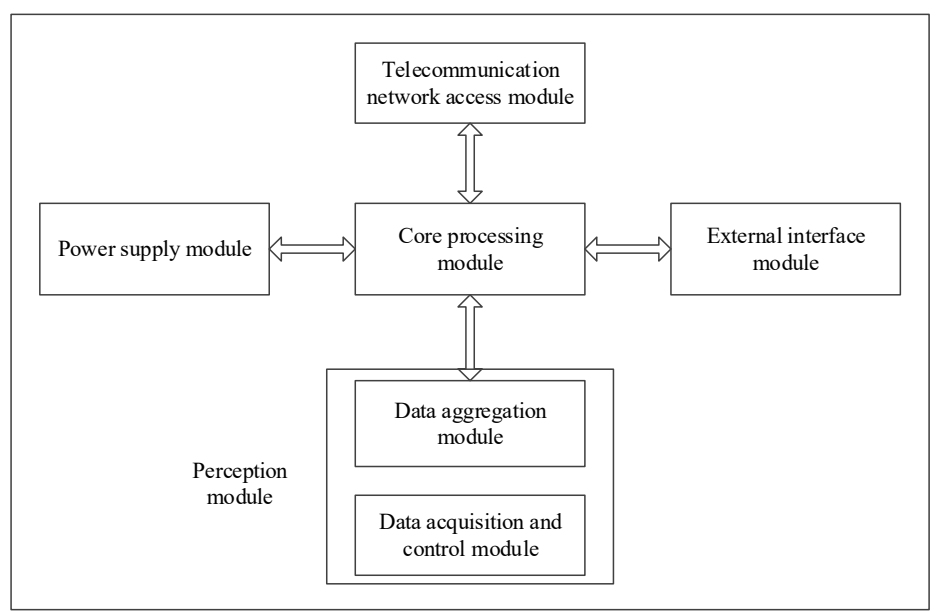

Figure 6. Terminal functional architecture of the Internet of Things

\subsection{Data Integration and Management}

From the perspective of data storage, traditional relational databases are not enough, because data comes from different sources, and data organization needs to be in multiple forms, such as documents, images, etc. From the perspective of data integration, traditional B2B data integration methods are not applicable to them. That's because such data generally cannot be organized by using the main database schema. The combination of these storage strategies usually depends on the type of data used and the custom view that is created on the basis of application requirements.

In addition, data persistence strategies are also necessary. This is because it is neither feasible nor necessary to store these large-scale, high-resolution data in many cases. Strategies involving data summarization and sampling, and corresponding source data storage are quite effective, especially when the data has a fairly high level of redundancy. Recall how sparse processing mode efficiently stores and processes data with a small amount of non-zero values in the case of high-dimensional data. These data structures are regular when provided with various analysis tools and libraries.

\section{Conclusion}

The integration of big data and the Internet of Things has brought unprecedented convenience to people's production and life. This article analyzes big data from four dimensions: data volume, data velocity, data diversity, and data value. It proves that the development trend of big data is larger in volume, faster in speed, more diverse, and more valuable, and its growth rate is fast and exponentially distributed. The Internet of Things is known as the third wave of the development of the world's information industry after the computer and the Internet. When the Internet of Things is integrated into big data, it will inevitably improve the intelligence level of human production and life. It can be applied to almost all aspects, but only the integration, management and technical framework in data processing are briefly introduced here.

\section{References}

[1]. Madakam S, Ramaswamy R, Tripathi S. Internet of Things (IOT): A Literature Review. Journal of Computer \& Communications, 2016, 03(5), pp.164-173.

[2]. Run H. The hardware design of the Internet of things gateway based on ARM, Nanjing: Nanjing University of Posts and Telecommunications, 2014, 57(3), pp. 190-191.

[3]. Tao T. What are the popular applications of big data. China Youth Daily.2014, 1(12), pp.179-192.

[4]. Maier S, Kenneth K. The big data age. Zhejiang: Zhejiang people's Publishing House.2013, 3(1), pp.32-35. 
[5]. Ma Y. The road to the Internet of things operation. Beijing: people post and Telecommunications Press, 2014, 1(3), pp. 65-72.

[6]. Lee J, Kao H. Service innovation and smart analytics for industry 4.0 and big data environment. Procedia CIRP, 2014, 16(3), pp13-18. 